\title{
Pour une approche conceptuelle de la notion de bonne administration
}

\author{
RHITA BOUSTA ${ }^{1}$
}

\section{RÉSUMÉ}

Depuis l'entrée en vigueur de la Charte des droits fondamentaux de l'Union européenne prévoyant un droit à une "bonne administration », cette expression se multiplie sans pour autant être définie. La bonne administration réunit de manière intuitive divers principes et droits préexistants à son apparition. Explicitant des réflexions à propos de quelques développements récents, cette recherche nous invite à une approche conceptuelle de la notion définie comme l'adaptation équilibrée des moyens de l'administration publique. Ouverte au débat, cette proposition de définition restrictive permet en outres de distinguer clairement bonne administration et bonne gouvernance.

Mots clef: Bonne administration, bonne gouvernance, principe(s) de bonne administration, droit à une bonne administration, pouvoir discrétionnaire, pondération, efficience, droit européen.

\section{Por una aproximación conceptual a la noción de buena administración}

\section{RESUMEN}

Desde la entrada en vigor de la Carta de los Derechos Fundamentales de la Unión Europea que proclama el derecho a una "buena administración",

1 Catedrática de la Universidad de Lille, Lille, Francia. Profesora de la Universidad Abierta de Cataluña (UOC), Barcelona, España. Doctora en Derecho de la Universidad París I, Panthéon-Sorbonne, París, Francia. Correo-e: rhita.bousta@univ-lille2.fr. Enlace ORCID: https://orcid.org/0000-0001-6589-7398. Fecha de recepción: 30 de julio de 2018. Fecha de modificación: 15 de agosto de 2018. Fecha de aceptación: 24 de septiembre de 2018. Para citar el artículo: BOUSTA, RITHA, "Pour une approche conceptuelle de la notion de bonne administration", Revista digital de Derecho Administrativo, Universidad Externado de Colombia, n. ${ }^{\circ} 21$, 2019, pp. 23-45. DOI: https://doi.org/10.18601/21452946.n21.04 
el uso de esta expresión se multiplica a pesar de no ser definida. La buena administración reúne intuitivamente diversos principios y derechos que existían antes de su aparición. Formulando sólidas reflexiones a la luz de algunas novedades, la presente investigación nos invita a una óptica conceptual y define la buena administración como la adaptación ponderada de los medios de la Administración pública. Abierta al debate, esta proposición de definición restrictiva permite distinguir la buena administración de otros conceptos, en particular la buena gobernanza.

Palabras clave: buena administración, buena gobernanza, principio(s) de buena administración, derecho a una buena administración, poder discrecional, ponderación, eficiencia, derecho europeo.

\title{
For a Conceptual Approach to Good Administration
}

\begin{abstract}
Since the enforcement of the Charter of Fundamental Rights of the European Union that provides a Right to "good administration", the term is often used and yet rarely defined. It is merely and intuitively reduced to a non-exhaustive list of pre-existing principles and rights. By presenting an in-depth analysis of recent legal developments, this paper claims for a conceptual approach to the good administration by defining it strictly as the proper and balanced use of the means of the public administration. Open to debate, this concept proposal helps to differentiate good administration from other concepts such as good governance.

Keywords: Good Administration, Good Governance, Principle(s) of Good Administration, Right to Good Administration, Discretionary Power, Balance, Efficiency, European Law.
\end{abstract}

\section{INTRODUCTION}

Depuis l'entrée en vigueur de la Charte des droits fondamentaux de l'Union européenne prévoyant un droit à "une bonne administration", l'usage de cette expression se multiplie. Et comme tout vocable en vogue, celui-ci coure le risque de se réduire à une coquille vide. Quelle que soit sa culture, le juriste est alors renvoyé à sa propre conception du Droit : se borner à retranscrire le droit positif, qui convertit très souvent la « bonne administration » en notion élastique et fonctionnelle, ou inclure d'autres sources de réflexion, telles que la doctrine, le droit souple ou encore le droit comparé afin de proposer, de manière inductive, une définition juridique de cette notion. 
Minoritaire $^{2}$, la seconde approche mérite à notre sens d'être creusée sans prétention d'exhaustivité ni même de justesse, et simplement afin d'ouvrir le débat sur le contenu à conférer, en droit, à la "bonne administration ». Si, fort heureusement, les réponses peuvent être différentes, les juristes de tout bord ont ici l'occasion de s'unir autour d'une quête de sens, même inachevée. C'est donc avec honneur que sur invitation de collègues colombiens, nous ouvrons ce numéro spécial de la Revista digital de Derecho Administrativo consacré à la buena administración.

Comme nous le montrions ailleurs ${ }^{3}$, la " bonne administration » est souvent réduite à un agrégat ouvert de droits et de principes, qui existaient bien avant l'apparition de ce vocable. En témoigne l'article 41 de la Charte des droits fondamentaux de l'Union européenne selon lequel le droit à une bonne administration comprend "notamment » ${ }^{4}$ les principes d'impartialité, d'équité, de délai raisonnable, de contradictoire, de transparence, de motivation, de réparation, et de communication dans une des langues des Traités. La «bonne administration » est alors au cœur des principes généraux du droit ${ }^{5}$ et, plus largement, de l'émergence d'un droit administratif européen ${ }^{6}$. Elle est exclusivement

2 Nous nous permettons de renvoyer à : RHITA BousTA, Essai sur la notion de bonne administration en droit public, Paris: L'Harmattan, coll. Logiques juridiques, 2010, p. 566.

3 Nous nous permettons de renvoyer à : RHITA BousTA, «Droit des étrangers - Mais à quoi sert le droit à une bonne administration ? ", Revue des droits de l'Homme, n. ${ }^{\circ} 12$, Avignon : OpenEdition, 2017, http://revdh.revues.org/3216; "Who said there is a right to good administration? A critical Analysis of art. 41 of the CFREu", European Public Law Review, vol. 19, n. ${ }^{\circ}$ 3, Alphen aan den Rijn: Kluwer Law International, 2013, pp. 481-488.

4 C'est bien l'adverbe employé par cet article : "Toute personne a le droit de voir ses affaires traitées impartialement, équitablement et dans un délai raisonnable par les institutions, organes et organismes de l'Union. 2. Ce droit comporte notamment : a) le droit de toute personne d'être entendue avant qu'une mesure individuelle qui l'affecterait défavorablement ne soit prise à son encontre ; b) le droit d'accès de toute personne au dossier qui la concerne, dans le respect des intérêts légitimes de la confidentialité et du secret professionnel et des affaires; c) l'obligation pour l'administration de motiver ses décisions. 3. Toute personne a droit à la réparation par l'Union des dommages causés par les institutions, ou par ses agents dans l'exercice de leurs fonctions, conformément aux principes généraux communs aux droits des États membres. 4. Toute personne peut s'adresser aux institutions de l'Union dans une des langues des traités et doit recevoir une réponse dans la même langue ». Nous surlignons.

5 JürGEN SCHWARZE, European Administrative Law, London: Sweet \& Marwell, 1992, pp. 1173. 1430 .

6 Jean-Bernard Auby et Jacqueline Dutheil de la Rochère (dirs.), Droit administratif européen, Bruxelles : Bruylant, 2007, pp. 235-241. V. aussi : Loïc Azoulal, « Le principe de bonne administration », dans Jean-Bernard Auby et Jacqueline Dutheil de la Rochère (dir.), Droit administratif européen, Bruxelles: Bruylant, 2007, pp. 493-518. 
conçue comme une "notion fonctionnelle ${ }^{7}$ visant à réunir des principes au cœur des transformations du droit administratif ${ }^{8}$ et du discours managérial ${ }^{9}$.

En France, beaucoup d'esprits la concevaient en dehors du domaine juri-

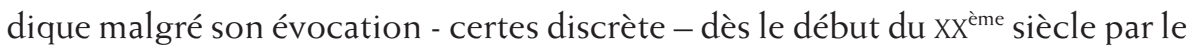
Doyen Hauriou ${ }^{10}$ et sa consécration relativement ancienne par certains droits étrangers. Cependant, cette expression a récemment fait son apparition dans une la loi de $2013^{[11]}$ retranscrite au nouveau Code des relations entre le public et l'administration de 2015 ${ }^{[12]}$. L'article L.231-5 de ce Code prévoit en effet la possibilité, "pour des motifs de bonne administration ", de déroger au principe selon lequel le silence administratif de plus de deux mois revêt une valeur d'acceptation de la part de l'administration. Ainsi, tout comme l'objectif de valeur constitutionnelle de bonne administration de la justice ouvrant des exceptions aux principes de répartition des compétences entre les juge administratif et judiciaire ${ }^{13}$, la " bonne administration » est à la fois indéfinie et réduite à une fonction de dérogation. Mais n'est-il pas critiquable qu'une notion flexible et polysémique se voit conférer un tel rôle ? La question de son contenu ne paraît à tout le moins pas dénuée d'intérêt.

D'un point de vue méthodologique, le droit comparé s'avère particulièrement utile ${ }^{14}$. Dès l'ouverture du recours contentieux dans les années trente, le juge grec employa l'expression en lui conférant, dans la majeure partie des

7 GeORGes VedeL, "La juridiction compétente pour prévenir, faire cesser ou réparer la voie de fait administrative", J.C.P., vol. I, n. ${ }^{\circ} 851$, Paris : CFJ, 1950. Le Doyen distingue alors les notions fonctionnelles des notions conceptuelles, qui ont un contenu matériel.

8 JaCQues CaIllosse, La constitution imaginaire de l'administration. Recherches sur la politique du droit administratif, Paris : P.U.F., 2008, pp. 396-401; LuCiano Parejo Alfonso, Crisis y renovación en el derecho público, Madrid: Centro de Estudios Constitucionales, 1991, p. 15.

9 JaCQues Chevallier et DANIÈLE LOSCHAK, " Rationalité juridique et rationalité managériale dans l'administration française ", R.F.A.P., n. ${ }^{\circ}$ 24, Paris : Documentation Française, 1982, pp. 53-94; Christopher Pollitt, Sandra van Thiel and Vincent Homburg, New Public Management in Europe. Adaptation and Alternatives, Basingstoke: Palgrave Macmillan, 2007, p. 229.

10 Selon le Doyen, le juge administratif est " chargé d'assurer la bonne administration » : MAURICE Hauriou, note sous C.E., 29 mars 1901, Sieurs Casanova, Canazzi et autres, rec. 333 ; S.1901, III, p. 73.

11 Loi n. ${ }^{\circ}$ 2013-1005 du 12 novembre 2013 modifiant l'article 21al9, phr. 1 de la loi n. ${ }^{\circ}$ 2000-321 du 12 avril 2000 relative aux droits des citoyens dans leurs relations avec les administrations.

12 Ordonnance n. ${ }^{\circ}$ 2015-1341 du 23 octobre 2015 : Décret n. ${ }^{\circ}$ 2015-1342 du 23 octobre 2015 .

13 Conseil constitutionnel, 23 janvier 1987, Loi transférant à la juridiction judiciaire le contentieux des décisions du Conseil de la concurrence, $n .{ }^{\circ} 86-224$ DC, cst. 16 ; art.L. 821-2 du code de justice administrative ; Conseil constitutionnel, 3 décembre 2009, n. ${ }^{\circ}$ 2009-595 DC (qualification d'objectif de valeur constitutionnelle).

14 LÉOnTIN-Jean CONSTANTINESCO, Traité de droit comparé. La méthode comparative, t. 2, Paris : L.G.D.J., $1974,{ }^{\circ}{ }^{\circ} 118$. 
cas, un sens proche de celui de bonne foi ${ }^{15}$. Développés après la Seconde Guerre mondiale, les principes de bonne administration figurent aussi dans la loi hollandaise de 1954 relative à la procédure administrative générale ${ }^{16}$ et furent réaffirmés dans les lois générales de 1992 et de $1994^{[17]}$. Mais ici encore, la conception est extensive : interdiction du détournement de pouvoir, diligence, motivation des actes administratifs, égalité, etc. Les ordres juridiques finlandais ${ }^{18}$ ou encore italien ${ }^{19}$ envisagent aussi la " bonne administration » en ce sens. En Espagne, la jurisprudence administrative a souvent recours au(x) principe(s) de bonne administration ou au droit à une bonne administration de la Charte européenne ${ }^{20}$. On pouvait aussi lire, dès l'exposé des motifs de la loi relative à la juridiction administrative de 1956, que les « infractions administratives » ne constituent pas seulement des préjudices pour les administrés, mais aussi des "entraves à une bonne et juste administration " ${ }^{21}$. On retrouve aussi l'expression dans certains Statuts autonomes comme ceux de Catalogne ${ }^{22}$ ou $\mathrm{d}^{\prime}$ Andalousie ${ }^{23}$. Le terme de «maladministration ${ }^{24}$ provient quant à lui du droit anglais et s'insère dans la réflexion sur la "bonne administration ", même si, selon nous, les deux termes ne sont pas des antonymes ${ }^{25}$. De plus, le juge

15 Georges Dellis, « Le principe de bonne administration dans la jurisprudence du Conseil d'Etat hellénique ", in Mélanges en l'honneur de Epaminondas P. Spiliotopoulos. Etat-loi-administration, Bruxelles : Bruylant, 1998, p. 31, note infrapaginale n. ${ }^{\circ} 1$.

16 Art. 5 de la loi du 16 septembre 1954 (Wet administratieve rechtspraak bedriffsorganisatie).

17 A ce sujet, v. CONSTANTIN KorTMAnN, « Les principes généraux du droit dans le droit public néerlandais ", in Christian-Albert Garbar (dir.), Mélanges en l'bonneur de Benoît Jeanneau. Les mutations contemporaines $d u$ droit public, Paris : Dalloz, 2002, pp. 147-159.

18 L'article 21 de la Constitution finlandaise dispose en effet que «les autres garanties d'une procédure équitable et d'une bonne administration, sont garanties par la loi ». Pour un aperçu des différentes lois générales administratives de bonne administration dans les pays scandinaves : KIRSI KuUsIKKO, "Advice, Good Administration and Legitimate Expectations: Some Comparative Aspects", European Public Law, September 2001, vol. 7, n. ${ }^{\circ} 3$, Alphen aan den Rijn: Kluwer Law International, 2001, pp. 455-472.

19 V. notamment : Guido Falzone, Il dovere di buona amministrazione, Milan: Dott. A. Giuffrè, 1953, p. 161.

20 V. notamment : Juli PONCE SolÉ, Deber de una buena administración y derecho al procedimiento administrativo y del ejercicio de la discrecionalidad, Valladolid: Lex Nova, 2001, p. 862; BEATRIZ TOMÁs MALLÉn, El derecho fundamental a una buena administración, Madrid: Instituto Nacional de Administración Pública, 2004, p. 342.

21 "Las infracciones administrativas se muestran realmente no tan solo como una lesión de las situaciones de los administrativos, sino como un entorpecimiento a la buena y recta administración".

22 Article 30 de la Loi organique n. ${ }^{\circ}$ 6/2006 du 19 juillet 2006, Ley Orgánica de Reforma del Estatuto de Autonomía de Cataluña, B.O.E. n. ${ }^{\circ} 172$ du 20 juillet 2006, "Derecho de acceso a los servicios públicos y a una buena Administración".

23 Loi organique n. ${ }^{\circ}$ 2/2007 du 19 mars 2007, Ley Orgánica de Reforma del Estatuto de Autonomía para Andalucía, B.O.E. n. ${ }^{\circ} 68$ du 20 mars 2007.

24 Parliamentary Commissioner for Administration Act, 22 mars 1967; Parliamentary and Health Commissioners Act, 15 mai 1987.

25 RHITA BousTA, Essai sur la notion de bonne administration en droit public, op. cit., n. ${ }^{\circ} 208$ et s. 
anglais s'est déclaré compétent pour se prononcer sur les cas de maladministration décelés par l'Ombudsman ${ }^{26}$ et, par ailleurs, évoque parfois les " principles of good administration $\aleph^{27}$ au regard duquel s'effectue le contrôle juridictionnel de l'administration britannique (judicial review ${ }^{28}$ ) sans toutefois inférer de la seule «bonne administration » une quelconque règle de droit indépendante et autonome $»^{29}$.

La notion voyage jusqu'en Amérique latine. À titre d'exemple, elle a tout récemment fait son apparition dans la Constitution de l'État de Mexico parmi les "facultés et obligations " ${ }^{30}$ du Gouverneur de cet État dans le cadre de sa gestion des finances publiques, et les contributions de ce numéro spécial permettront sans doute d'illustrer les différentes acceptions de la «buena administración » en Colombie.

Pour certains, l'hétérogénéité des manifestations de la « bonne administration » rendrait vaine toute recherche de sa consistance ${ }^{31}$. Sa flexibilité invite en effet, a priori, à l'envisager comme un standard indéfini du droit public ${ }^{32}$. Mais à notre sens, ces usages extensifs ne sont pas un obstacle à sa conceptualisation. À l'inverse, ceux-ci sont tout autant d'outils - voire d'incitations ! - de "fabrication » de la notion. C'est en tous les cas la démarche que nous adoptons depuis une dizaine d'années.

Dans la continuité de nos précédents travaux, et dans l'optique des contributions de ce Numéro spécial, nous souhaiterions ici expliciter notre définition (1) et rappeler qu'à notre sens, la bonne administration se distingue d'autres concepts desquels elle est souvent confondue ou associée, principalement la bonne gouvernance (2).

26 Nous nous permettons de renvoyer à RHITA BousTA, "Contribution à une définition de l'Ombudsman ", RFAP, n. ${ }^{\circ}$ 123, Paris : École nationale dradministration, 2007, pp. 387-397.

27 Pour exemple : R v. Panel on Take-overs \& Mergers, ex parte Datafin plc [1987] 2 W L.R 699, CA.

28 Lord DipLOCK, in Council of Civil Service Union v. Minister for the Civil Service, [1985] A.C. 374 Ce contrôle renferme globalement trois aspects : la compétence (legality), la procédure (procedural propriety) et la rationalité (rationality).

29 Lord BRIDGE, in H.L. [1983] 1 All E.R. 439-446; Lord MCNEILL, in R v. Secretary of State for the Environment, ex parte Birminghan CC CO/72/87; Lord BINGHAM, in Khan $v$. Secretary of State for Home Department CA [1987] Imm. AR 543.

30 Constitución Política del Estado Libre y Soberano de México, 17 novembre 1917 (réforme du 3/02/2017), cap. III, sección segunda, art. 77, XXI: "Cuidar la recaudación y buena administración de la Hacienda Pública del Estado".

31 Pour exemple : A. J. Callaghan, "Maladministration", The Ombudsman Journal, n. ${ }^{\circ}$ 7, Vienna: International Ombudsman Institute, 1988, p. 31.

32 STÉPHANE RIALS, Le juge administratif français et la technique du standard. Essai sur le traitement juridictionnel de l'idée de normalité, t. 135, Paris : L.G.D.J., 1980, p. 564. 


\section{PROPOSITION DE DÉFINITION DE LA NOTION DE BONNE ADMINISTRATION}

L'adjectif «bon », ainsi que ses relations avec le "Bien », renvoient d'abord à des considérations philosophiques et morales $^{33}$. Socrate affirmait ainsi que la détermination du "vrai » par la science était subordonnée à une fin morale, à savoir le "Bien " ${ }^{34}$. Sous la plume de Jérémie Bentham ${ }^{35}$, le «bon " est relié au domaine de la déontologie, lui-même empreint de considérations morales. Cependant, nous pensons que la «bonne administration » peut aussi revêtir un sens juridique propre.

Nous avons ainsi défini ailleurs ${ }^{36}$ la bonne administration comme l'adaptation équilibrée des moyens de l'administration publique. Il s'agit là d'un essai, d'une proposition, qui doit être explicitée car elle sous-tend une analyse critique de l'approche plus répandue, fonctionnelle et flexible de la "bonne administration ». Il convient donc de revenir sur les deux critères cumulatifs, consubstantiels et exclusifs de la notion proposée : l'adaptation des moyens (1.1) ainsi que leur pondération (1.2).

\subsection{UNE ADMINISTRATION AUX MOYENS ADAPTÉS ET OPTIMISÉS}

L'idée d'adaptation est partie intégrante de l'administration, qui signifie : service (ministratio) pour (ad). L'étymologie de ce terme aide à comprendre que la bonne administration désigne le bon usage des moyens de l'administration. Cette remarque est confirmée par la définition du «bon » livrée par l'Académie française : ce qui est « conforme à sa nature, à sa destination; qui convient bien à son usage, qui remplit bien sa fonction, [...] qui est approprié, adapté au but recherché " ${ }^{37}$ (1.1.1). Il nous semble alors évident que la bonne administration se situe au cœur du pouvoir discrétionnaire de l'administration publique (1.1.2).

\subsubsection{La focalisation sur les moyens de l'administration}

On retrouve les prémices de cette focalisation sur les moyens sous la plume $\mathrm{d}^{\prime}$ Aristote $^{38}$. Si l'éminent philosophe reprend la distinction platonicienne entre

33 Aristote, Éthique à Nicomaque, Paris : J. Vrin, 1987, livre X, 10, spéc. 1181 b.

34 JaCques Chevalier, La notion du nécessaire chez Aristote et chez ses prédécesseurs, Paris : Librairie Félix Alcan, 1915, p. 41.

35 Jérémie Bentham, Déontologie ou science de la morale, Paris ; Charpentier, 1834, p. 359.

36 RHITA BousTA, Essai sur la notion de bonne administration en droit public, op. cit., pp. 109-160.

37 Académie Française, Dictionnaire de l'Académie française, fasc. II, 9. éd., Paris : Imprimerie nationale, 1987, p. 151.

38 Aristote, op. cit., pp. 146-150. 
les moyens et la fin, il en inverse le rapport de subordination ${ }^{39}$. Le " nécessaire ", qui révèle un lien $\mathrm{d}^{\prime}$ " inbérence causale » ${ }^{40}$, constitue l'objet même de la science. C'est alors la fin qui est subordonnée aux moyens : "le bon choix ne se mesure plus à la rectitude de l'intention, mais [aux] moyens »" ${ }^{41}$. Le " bon » s'oppose alors au «bien », qui concerne les fins poursuivies et la volonté. Contrairement à celle-ci, purement morale, le choix est technique.

Ainsi, selon Nieto, une bonne organisation administrative s'adapte aux nécessités sociales qui l'orientent vers l'intérêt général ${ }^{42}$. C'est aussi l'idée d'adaptation des moyens qui est au cœur de la notion anglaise de « administrative good behavior ${ }^{43}$.

Il convient dès lors de différencier l'adaptation des moyens de leur efficacité, qui mesure un résultat obtenu au regard du résultat escompté. Par contraste, la notion de bonne administration n'est centrée ni sur les résultats ni sur la fin de l'administration, mais sur ses moyens, bien que ces éléments soient incontestablement liés. Les moyens de l'administration comprennent en outre sa méthode, la notion de bonne administration renvoie donc à un procédé adapté et équilibré permettant de répondre à un objectif. C'est ainsi que la procédure administrative non contentieuse peut être envisagée comme un moyen de prendre un acte administratif.

La théorie de l'administration doit alors déterminer les facteurs de cette optimisation des moyens, au premier rang desquels figure la compétence des administrateurs. Ici, le «bon administrateur » est l'incarnation personnifiée de l'idée d'adaptation des moyens. Ainsi que l'a rappelé le Tribunal de première instance des Communautés européennes, l'examen préalable des mérites des candidats au sein de chaque direction générale «participe du principe de bonne administration $»^{44}$.

L'adaptation des moyens financiers est aussi au cœur de la « bonne administration ». L'efficience désigne ainsi l'accomplissement d'un objectif au moindre coût financier. Elle implique donc une mise en balance entre les moyens (inputs) et l'objectif (output). Alors que l'efficacité vise le rapport entre les résultats attendus et les résultats obtenus, l'efficience est évaluée sur la «base d'un calcul d'optimisation visant à minimiser les coûts $»^{45}$. Or, la gestion des deniers publics est désormais dominée (du moins dans les esprits) par une prise en compte du

Pierre Aubenque, La prudence chez Aristote, Paris : P.U.F., 1997, pp. 134-135.

JaCques Chevalier, La notion du nécessaire chez Aristote et chez ses prédécesseurs, op. cit., p. 1.

Pierre Aubenque, op. cit., p. 122.

Alejandro Nieto, La "nueva" organización del desgobierno, 3. a ed., Barcelona: Ariel, 2003, p. 147.

Herbert A. Simon, Administrative Bebavior. A Study of Decision-Making Process in Administrative Organization, 2. ${ }^{a}$ ed., New York: The Free Press, 1957, p. 39. 
rapport moyen / fin. Dès lors, une précision s'impose : la pure véracité des comptes n'est ici pas concernée. L'efficience n'est en effet pas l'antonyme de la corruption qui a trait davantage, comme nous le verrons, à la mauvaise gouvernance. Le noyau dur de la notion de bonne administration n'inclut pas, à notre sens, la transparence, car elle désigne avant tout un rapport qualitatif, à savoir la manière optimale dont a été utilisé l'outil financier.

La notion de bonne administration vise donc, à notre sens, la meilleure réalisation d'un objectif par l'optimisation des moyens de l'administration. La notion étudiée ne peut être comprise qu'en adoptant une vision fonctionnelle de l'administration. C'est en effet dans son action, c'est-à-dire dans la concrétisation matérielle de sa fonction, que l'administration se révèle " bonne » ou " mauvaise ". "La bonne ou manvaise administration ne doit pas être définie en termes absolus via la relation objectif-moyen-résultat, mais via une relation plus complexe objectif-moyenmanière ['modos']-résultat $»^{46}$. On est alors au cœur du pouvoir discrétionnaire.

\subsubsection{La bonne administration, au cœur du pouvoir discrétionnaire}

Le pouvoir discrétionnaire permet à l'administration d'accomplir ses objectifs avec une certaine marge de manœuvre et d'opter pour " le choix le plus adéquat, la meilleure des solutions possibles ${ }^{47}$. La notion de bonne administration est donc parfois associée à la "bonne utilisation du pouvoir discrétionnaire ${ }^{48}$ ou encore au "meilleur usage du pouvoir discrétionnaire " ${ }^{49}$. L'Ombudsman, chargé de garantir la bonne administration, doit remédier à la "'manvaise' utilisation du pouvoir discrétionnaire » de l'administration publique ${ }^{50}$. En effet, le choix de la meilleure solution possible relève du "devoir juridique de bonne administration " " $^{51}$. L'administration se doit d'apprécier et de jauger, face à chaque situation concrète et spécifique, "la solution la plus adéquate et conforme à l'intérêt public " ${ }^{52}$. En somme, les principes

46 Marcos Gómez Puente, La inactividad de la Administración, 3. ${ }^{a}$ ed., Madrid: Aranzadi, 2002, p. 62.

47 "Permitir a la Administración la elección de la más adecuada, la mejor de las soluciones posibles": GeRMán FERNÁNDEZ FARRERES, La subvención: concepto y régimen jurídico, Madrid: Instituto de Estudios Fiscales, 1983, p. 642.

48 Eduardo García de EnTerría, "La interdicción de la arbitrariedad en la potestad reglamentaria", R.A.P., n. ${ }^{\circ}$ 30, Madrid: Centro de Estudios Políticos y Constitucionales, 1959, p. 148.

49 Doña María José Alonso Mas, La solución justa en las resoluciones administrativas, Valencia: Tirant lo Blanch, 1998, pp. 374 et s.

50 Álvaro Gil-Robles y Gil-Delgado, "La filosofía del defensor del Pueblo", in Paloma Biglino (dir.), El procurador del común. Defensor del Pueblo y Comunidades Autónomas, Valladolid: Cortes de Castilla y León, 1995, p. 23.

51 Doña María José Alonso Mas, op. cit., pp. 35 et 231.

52 "Deber de apreciar y valorar ante cada situación concreta y especifica la solución más adecuada y conforme al interés público": Germán FeRnández FARRERES, op. cit., p. 641. 
de bonne administration ont " changé la façon dont le pouvoir discrétionnaire était considéré d'un point de vue juridique $»^{53}$.

Ainsi, dans l'arrêt Commune de Fontenay-sous-Bois ${ }^{54}$ où le Conseil d'Etat évoque pour la seule fois, à notre connaissance, la « bonne administration » en dehors du droit subjectif du même nom provenant du droit européen, celle-ci permet d'aménager le découpage cantonal afin d'amoindrir les disparités quantitatives de population en milieu urbain. Selon les commentateurs, en imposant une cohérence et une simplicité administrative dans le découpage des circonscriptions ( «bonne administration »), le Conseil d'État contrôle le pouvoir discrétionnaire. Il est en effet clair que la notion de bonne administration encadre la manière dont il est utilisé. Il faut donc relever le lien unissant la bonne utilisation du pouvoir discrétionnaire à la notion de bonne administration. Tout comme cette dernière, le premier est intrinsèquement lié à l'idée d'optimisation. Cette adaptation optimale des moyens au regard de l'objectif poursuivie n'est cependant pas absolue : elle doit être conciliée avec d'autres éléments.

\subsection{UNE ADAPTATION ÉQUILIBRÉE DES MOYENS}

L'essence du droit administratif réside dans le maintien de la conciliation entre les intérêts de l'État et ceux des citoyens. Jean-Marie Pontier retrace ainsi le passage d'un droit de dérogation à un droit de confrontation des droits pour aboutir à ce droit d'équilibre ${ }^{55}$. La notion de bonne administration s'inscrit dans cette troisième étape. À cette fin, elle concilie des éléments en conflit (1.2.1) et contrairement à une idée reçue, celle-ci ne désigne à notre sens pas la satisfaction optimale et unilatérale des citoyens (1.2.2).

\subsubsection{La conciliation d'intérêts en conflit}

La notion de bonne administration renferme l'idée d'équilibre entre différents intérêts ou différents principes. En premier lieu, elle unifie les principes de bonne administration consacrés par le droit positif. D'abord, en leur conférant un but commun, à savoir à notre sens l'adaptation : ensuite, en les conciliant entre eux.

En second lieu, elle harmonise les différents intérêts, qui sont davantage imbriqués que conflictuels. Dans un État « démytbifié » ${ }^{56}$, la vision dichotomique

53 W. N. de Waard Boudewijn et Adrienne J. C. de Moor-van Vugt, « Les changements récents dans le droit administratif néerlandais », in Gérard Marcou (dir.), Les mutations $d u$ droit de l'Administration en Europe. Pluralisme et convergences, Paris: L'Harmattan, 1995, p. 229.

54 C.E., ass., 18 novembre 1977, n. ${ }^{\circ} 01967$; Michèle Nauwelaers et Olivier Dutheillet de LAMOTHE, A.J.D.A., chron., Paris: Dalloz, 1978, pp. 209-210.

55 Jean-Marie Pontier, "Qu'est-ce que le droit administratif ? ", A.J.D.A., n. ${ }^{\circ} 35$, Paris : Dalloz, 2006, pp. 1937-1940.

56 Jacques Chevallier, L'État post-moderne, 2. éd., Paris : L.G.D.J., 2004, p. 65. 
et hiérarchisée administré(s) / Administration semble en effet dépassée. "Intérêt public » et " intérêt privé » ne doivent pas être opposés de manière stricte. La Constitution espagnole évoque par exemple les « intérêts généraux » ${ }^{57}$, mettant en exergue la multiplicité des expressions de l'intérêt général qui peuvent entrer en conflit ${ }^{58}$. La même idée est clairement exprimée par le juge européen selon lequel le principe de bonne administration impose à l'administration d'opérer une mise en balance des intérêts en cause ${ }^{59}$.

En creux, Alberto Pérez Calvo définit la " mauvaise administration " comme le dysfonctionnement des procédures administratives résultant d'une mauvaise pondération des intérêts en cause ${ }^{60}$. Au sujet de la maladministration, K. C. Wheare affirme que l'administration doit non seulement être efficace en atteignant les objectifs d'une politique sans retard, mais aussi assurer, de manière raisonnable, l'équilibre entre l'intérêt public qu'elle défend et l'intérêt privé qu'elle limite ${ }^{61}$.

La bonne administration désigne le contrepoids entre deux types d'intérêt : celui de l'individu ("dignitary rationale ") et celui de l'administration ("instrumentary rationale »). Or, «un effacement démesuré du pouvoir de l'administration en faveur de la protection de l'individu et de la transparence est incompatible avec la 'bonne administration' ${ }^{62}$. L'institution de l'Ombudsman démontre avec vigueur cette idée. Garant de la bonne administration, celle-ci est en effet considérée comme un conciliateur (médiateur) d'intérêts en conflit. Le passage de cas individualisés de mauvaise administration à la généralisation en termes de bonne administration en témoigne. La notion de bonne administration ne saurait ainsi être confondue avec la satisfaction unilatérale des citoyens.

\subsubsection{La notion de bonne administration ne traduit pas exclusivement l'optique du citoyen}

Instinctivement, l'assimilation entre la notion de bonne administration et la satisfaction unilatérale de l'administré ou du citoyen paraît tentante. La " bonne administration » est dans ce cas souvent réduite à un "slogan».

57 Article 103.1.

58 GÉRARD MARCOU, « La Gouvernance : innovation conceptuelle ou artifice de présentation?", Annuaire 2006 des Collectivités locales : La gouvernance territoriale, Paris : C.N.R.S., 2006, p. 12.

59 T.P.I.C.E., $4^{\text {e }}$ ch., 9 juillet 1999, New Europe Consulting, préc., pt. 39.

60 Alberto Pérez Calvo, "Aspectos constitucionales del Defensor del Pueblo", Revista del Departamento de Derecho Político, n. ${ }^{\circ} 4$, Madrid: UNED, 1979, p. 90.

61 Keneth Clinton Wheare, Maladministration and its remedies, London: Stevens \& Sons, 1973, p. 20.

62 "Disproportionate curtailing of the administration for the sake of individual protection and transparency is to be regarded as incompatible with 'good administration'": HANNS PETER NEHL, Principles of Administrative Procedure in EC Law, Oxford: Hart Publishing, 1999, p. 167. 
Cette démarche, utile pour certaines revendications sociales ou politiques, a toutefois des limites conceptuelles.

Elle semble en réalité découler de certaines classifications des principes du même nom. Par exemple, Diana Woodhouse distingue les principes de bonne administration se rattachant à l'intérêt de l'individu ("individual interest ») des principes de bonne administration servant l'intérêt public ( " public interest ») ${ }^{63}$. Relèverait de la première catégorie les principes suivants : motivation, transparence, confidentialité, équité, confiance légitime. L'intérêt public serait, lui, garanti notamment par la rapidité et l'efficience des décisions. Cette classification n'est toutefois pas exempte de critiques. Les principes censés servir l'intérêt de l'administré peuvent également satisfaire l'intérêt public, et inversement. Comme cela a été montré, il semble au contraire que la notion - qui doit être différenciée du ou des principe(s) - de bonne administration réalise l'équilibre entre ces deux optiques; elle ne renferme donc qu'une seule acception.

Les rapports des Ombudsmän militent en ce sens. Le pouvoir d'auto-saisine montre en effet que la bonne administration ne découle pas uniquement du point de vue de l'administré. Dans certains État, tels que la Suède, le Portugal ou encore la Finlande, l'intérêt pour agir n'est d'ailleurs pas requis ${ }^{64}$. Un manque de personnel dans l'administration de la justice (adaptation des moyens humains) concerne à la fois l'intérêt du plaignant et le fonctionnement général du système administratif ${ }^{65}$. De même, l'impératif de délai raisonnable sert aussi bien les intérêts de l'administré que ceux de l'administration ${ }^{66}$. Ainsi, à l'occasion de l'arrêt Stark, la Cour de justice de l'Union européenne rappelle l'obligation incombant non pas à l'administration, mais au plaignant de prendre des mesures appropriées afin que sa convocation lui parvienne à son lieu de séjour ${ }^{67}$.

En somme, la notion de bonne administration vise l'adaptation des moyens obtenue par une pondération raisonnable et raisonnée des éléments pertinents. L'idée d'équilibre lui est, à notre sens, consubstantielle. Cette définition centrée sur les moyens, permet, en outre, de distinguer la notion de bonne administration de la bonne gouvernance avec laquelle elle est souvent confondue.

63 Diana Woodhouse, In Pursuit of Good Administration. Ministers, Civil Servants and Judges, Oxford Clarendon Press, 1997, p. 190.

64 Jacob Söderman, " Le citoyen, l'administration et le droit communautaire ", R.M.U.E., n. ${ }^{\circ}$ 2, Paris : Clement Juglar, 1998, p. 48.

65 Defensor del Pueblo, Rapport annuel de 2004, présenté au Parlement en avril 2005, Madrid: éd. Publicaciones del Congreso de los Diputados, 2005, p. 148.

66 C.J.C.E., $1^{\text {e }}$ ch., 4 février 1970, Van Eick c/ Commission, aff. 13/69, rec.1970, p.3, pt.4.

67 C.J.C.E., $1^{\mathrm{e}}$ ch., 27 novembre 1986, Strack c/ Parlement européen, aff. 155/85, rec. 1986, p. 3561 


\section{LA DISTINCTION ENTRE BONNE ADMINISTRATION ET BONNE GOUVERNANCE}

La " bonne administration » et la " bonne gouvernance " doivent à notre sens être clairement distinguées. Si l'administration et le gouvernement se rapprochent d'un point de vue organique et organisationnel, l'administration exécutrice ne doit pas être confondue avec le gouvernement politique. En d'autres termes, l'intensité du lien s'affaiblit d'un point de vue fonctionnel.

Or, la bonne administration est souvent employée pour désigner des éléments se rattachant davantage, à notre sens, au bon gouvernement ou à la bonne gouvernance. Ailleurs, nous avons donc montré de manière approfondie la spécificité de la notion de bonne administration vis-à-vis d'autres notions (qualité, bonne administration de la justice, confiance légitime, etc.) ${ }^{68}$. Dans l'optique de ce Numéro spécial, il nous semble pertinent de centrer l'analyse sur la distinction entre bonne administration et bonne gouvernance. En effet, à notre sens, tant leur domaine (2.1) que leur objet ne coïncident pas (2.2).

\subsection{UNE DIFFÉRENCE DE DOMAINES}

Pour beaucoup, la gouvernance est réduite à un « bric-à-brac de dispositifs hétérogènes, instables et évolutifs " ${ }^{69}$ (2.1.1). Elle se distingue ainsi de la notion de bonne administration si tant est que l'on accepte la possibilité de définir la seconde de manière restrictive. De plus, sans nier son intérêt pour les réflexions sur le droit, il semble que la bonne gouvernance relève davantage du domaine politique ou économique que du domaine juridique (2.1.2).

\subsubsection{La bonne gouvernance, une notion large}

Équivalente de la notion anglaise de governance ${ }^{70}$, la gouvernance semble souvent évoquée sans véritable contenu matériel ${ }^{71}$. Elle appelle a priori et globalement au déploiement et à l'intensification de la concertation et de la négociation ainsi qu'à la transparence ${ }^{72}$. "Mot valise ${ }^{73}$, celle-ci se caractérise en réalité avant tout par sa polysémie.

68 RHITA Bousta, Essai sur la notion de bonne administration en droit public, op. cit., pp. 221-256.

69 Mireille Delmas-Marty, Les forces imaginantes du droit, t. 3, La Refondation des pouvoirs, Paris éd. du Seuil, 2007, pp. 69-97.

70 GÉRARD MarCOU, « Gouverner les villes par le droit ? », C.U.R.A.P.P., La gouvernabilité, Paris : P.U.F., 1996, pp. 181-185.

71 GerRy StOKER, "Cinq propositions pour une théorie de la gouvernance », Revue internationale des sciences sociales, n. ${ }^{\circ} 155$, Toulouse : ERES, 1998, p. 20.

72 Gérard Cornu, Vocabulaire juridique, 8. éd., Paris : P.U.F., 2007, p. 444

73 FRANÇOIS MORVAN, "Les aspects majeurs de la nouvelle gouvernance financière ", in Michel Bouvier (dir.), Réforme des finances publiques, démocratie et bonne gouvernance, Paris : L.G.D.J., 2004, p. 122. 
Il existe en effet, au-delà de l'utopie du " meilleur gouvernement $»^{74}$, une multiplicité de points de vue concernant la bonne manière de gouverner. La bonne gouvernance est alors fréquemment évoquée à travers une diversité de principes renvoyant à des réalités hétérogènes. À titre d'illustration, cinq principes dits de «bonne gouvernance » ont été énoncés à l'échelle européenne ${ }^{75}: l^{\prime}$ ouverture, la participation, la responsabilité, l'efficacité et la cohérence. Kenneth Stowe énonce également six critères de définition : la liberté politique (liberté d'expression et droit de vote) ; la protection judiciaire et constitutionnelle des droits individuels ; l'indépendance de la justice ; le maintien d'une monnaie stable ; le développement de la société par l'éducation et la santé ; et, enfin, la responsabilité de l'exécutif envers un parlement élu librement ${ }^{76}$. D'aucuns soulignent à ce sujet la nécessité d'un gouvernement local fort ${ }^{77}$. D'autre envisage la notion sous son aspect économique : celle-ci désignerait alors l'efficacité des remèdes institutionnels contre la corruption dans une économie de marché ${ }^{78}$.

Cette multiplicité d'idées rattachées à la «bonne gouvernance » témoigne de son élasticité. Il n'est de plus pas interdit de s'interroger sur son réel apport au regard de la notion de gouvernance. En effet, certains auteurs définissent cette dernière, en tant que forme spécifique de gouvernement, par les caractéristiques de la bonne gouvernance ${ }^{79}$. De même, la gouvernance a pu être définie autour de trois pôles rappelant davantage la problématique de la bonne gouvernance : le processus de rationalisation des choix ou des objectifs politiques, l'instauration de dispositifs d'échanges et de négociation, et la participation ${ }^{80}$.

Les exemples illustrant la polysémie des notions de gouvernance et de bonne gouvernance pourraient être multipliés à loisir. Dans l'optique choisie, il faut se limiter à la distinguer de la bonne administration. De plus, la bonne gouvernance relève avant tout, sans y être restreinte, du domaine politique.

74 ThOmas More, Le traité de la meilleure forme de gouvernement ou l'Utopie, Genève : Librairie Droz, 1983 [1936], p. 153.

75 Commission des Communautés EuropéEnNES, Gouvernance européenne, un Livre blanc, 25 juillet 2001, COM (2001) 428 final. Ce livre blanc est souvent considéré comme le fondement des principes de bonne administration : JeAn-Bernard Auby et JaCQueline DutheIl DE LA ROCHËRE, op. cit., p. 379.

76 Kenneth Stowe, "Good Piano won't play Bad Music: Administrative Reform and Good Governance", Public Administration, vol. 70, Nueva Jersey: Wiley-Blackwell, 1992, p. 388.

77 ARnaud Cabanes, Essai sur la gouvernance publique. Un constat sans concession... quelques solutions sans idéologie, Paris: Gualino, 2004, pp. 30-39, spéc. p. 29.

78 Mark Bevir, "What is Governance", in Mark Bevir (dir.), Public Governance, t. 1, London: Sage Publication, 2007, pp. vi-xlv.

79 Gary StOcker, op. cit., p. 19.

80 C.U.R.A.P.P., La gouvernabilité, Paris: P.U.F., 1996, pp. 11-13. 


\subsubsection{La nature politique de la bonne gouvernance}

La question de la bonne gouvernance est fréquemment associée à celle de la corruption politique ou économique ${ }^{81}$. Il est souvent question de transparence.

À titre d'exemple, dans le sommaire du Rapport intitulé "Renforcement de la bonne gouvernance en Afrique ${ }^{82}$, il est question de politique, de conflits ou encore de corruption. Les facteurs d'une bonne gouvernance sont évoqués de la manière suivante : l'élection des dirigeants, leur responsabilité politique, leur attention aux besoins de la population, le lien de réciprocité, la définition du paradigme économique, le climat des affaires responsable et transparent, la capacité de l'État à innover, etc. Il en est de même d'un dossier relatif à la "gouvernance mondiale ${ }^{83}$ qui renvoie à la mondialisation économique ou encore à l'aide humanitaire.

Il convient donc d'insister sur l'importance de la valeur " corruption " dans l'appréhension de la bonne gouvernance : responsabilité, transparence, honnêteté et démocratie ${ }^{84}$. C'est ainsi que l'on évoque aussi parfois la «nouvelle gouvernance ${ }^{85}$ définie autour de trois critères fondamentaux : la responsabilité civique collective et individuelle des membres de l'organisation ; la concertation et le partage des pouvoirs et des responsabilités à plusieurs échelons, enfin, la perspective systémique, c'est-à-dire la compréhension des interactions entre acteurs et leur influence efficace sur la réalité.

La parcimonie des considérations juridiques entourant la gouvernance, qui se caractérise davantage par sa dimension politique est donc peu contestable. La gouvernance "se situe 'en marge ou à la marge' du droit $»^{86}$ et a pour fonction de justifier les politiques publiques. Parfois, l'accent est davantage mis sur l'aspect économique et social de la (bonne) gouvernance. C'est l'optique logiquement adoptée par la Banque mondiale en 1992 ${ }^{[87]}$. Suivant le contexte et la prétendue " avancée démocratique ", la bonne gouvernance est synonyme d'anti-corruption,

81 Jean-Pierre Gaudin, Pourquoi la gouvernance? Paris : Presses de science politique, 2002, p. 137.

82 Banque Africaine du Développement, Renforcement de la bonne gouvernance en Afrique, Paris: Économica, 2001.

83 "Gouvernance mondiale : la nécessaire utopie ", Revue politique et parlementaire, avril-juin 2005, vol. 107, n. ${ }^{\circ} 1035$, pp. 1-190.

84 Martin Minorgue, "Is Good Governance a Universal Value?", Public Policy and Management: Working Paper Series, n. ${ }^{\circ}$ 6, Manchester: University of Manchester, 1998, p. 4.

85 Françols Morvan, op. cit., pp. 121-134.

86 Constantin A. StePhanOu, " La nouvelle gouvernance européenne : bilan et perspectives », Cabiers de droit européen, vol. 42, n. ${ }^{\circ}$ 5-6, Brussels : Léon Goffin, 2006, p. 582.

87 BANQUE Mondiale, Governance and Development, Washington, mai 1992, 61 p. Rapport disponible sur : http://documents.worldbank.org/curated/en/604951468739447676/Governanceand-development [consulté le 31/07/2018]. 
d'efficience ou de gestion efficace des fonds. Pour certains ${ }^{88}$ alors, la « nouvelle gouvernance » désigne le modèle de gestion publique inspiré des entreprises. La bonne gouvernance financière permettrait une nouvelle organisation de la structure et des méthodes de la finance internationale.

La notion de bonne gouvernance est donc plus large - pour ne pas dire indéfinie - que l'acception que nous proposons de la bonne administration. Ces deux notions se différencient aussi, et principalement, par leur objet.

\subsection{UNE DIFFÉRENCE D'OBJETS}

La bonne administration vise l'administration publique ; la bonne gouvernance, quant à elle, concerne principalement le gouvernement (2.2.1). De plus, alors que, comme nous l'avons vu, la bonne administration a trait exclusivement aux moyens de l'administration, la bonne gouvernance concerne davantage ses objectifs ou ses résultats (2.2.2).

\subsubsection{Gouvernement (bonne gouvernance) et administration (bonne administration)}

La bonne gouvernance concerne principalement trois acteurs : l'État, la société civile et le secteur privé ${ }^{89}$. Ont pu être évoqués à ce propos les «niveaux multiples du principe de bonne gouvernance ", à savoir le législateur, le juge et l'administration alors que la notion de bonne administration se situe à un " niveau unique ${ }^{90}$. Bien qu'elle ne se réduise pas à ce dernier, la bonne gouvernance souligne la nécessaire distinction (qui n'efface en rien leurs liens ténus) entre le gouvernement et l'administration. Elle concerne le gouvernement dans son exercice de la politique ${ }^{91}$ et a trait à la légitimité et à la bonne exécution du pouvoir par l'État. La gouvernance se substitue ainsi aux termes de " gouvernement " dans le secteur public et de "gestion » dans le secteur privé. Elle est en outres fondée sur les idées de légitimité, de liberté des individus, de participation citoyenne et d'État de droit.

En revanche, la notion de bonne administration a pour objet exclusif l'administration publique. Toute considération d'ordre politique doit être exclue, en

88 Michel Bouvier, "Avant-propos », in Michel Bouvier (dir.), Réforme des finances publiques, démocratie et bonne gouvernance, Paris : L.G.D.J., 2004, p. 3.

89 Ali Farazmand, "Sound Governance in the Age of Globalisation: A Conceptual Framework", in Ali Farazmand (dir.), Sound Governance, London: Praeger, 2004, pp. 1-25.

90 Julie LASSALLE, Le principe de bonne administration en droit communautaire, Thèse droit public, Paris Université de Paris II, 2008, pp. 652-657.

91 FRANCISCO URÍA FERNÁNDEZ, "El buen gobierno en al ámbito del sector público. Una reflexión jurídica", en Manuel Balmaseda y Melguizo et al. (dir.), La Administración pública que España necesita, Madrid: Círculo de Empresarios, 2007, pp. 339-340. 
particulier lorsqu'il s'agit d'en faire une notion juridique. Ainsi, l'objet de cette dernière notion est plus restreint que celui de la bonne gouvernance.

Les principes de bonne gouvernance sont donc une combinaison de principes de bonne administration (efficacité, efficience, légalité) et des idées de démocratie ou d'éthique ${ }^{92}$. On peut d'ailleurs regretter le changement opéré par les rédacteurs de la Charte des droits fondamentaux de l'Union européenne, substituant le terme de "bonne administration " à celui de "bonne gouvernance ${ }^{93}$. Cette différenciation est aussi implicitement confortée lorsque la bonne administration est qualifiée de composante de la bonne gouvernance.

Outre leur champ d'application, l'optique des notions de bonne administration et de bonne gouvernance constitue un critère supplémentaire de leur distinction. Plus précisément, alors que la notion de bonne administration est centrée, comme nous l'avons vu, sur les moyens de l'administration, la notion de bonne gouvernance s'intéresse à ses buts, voire à ses résultats.

\subsubsection{Analyse des moyens (bonne administration) et analyse des résultats et des buts (bonne gouvernance)}

Comme nous le suggérions plus haut, la bonne administration a pour objet les moyens de l'administration, et désigne leur adaptation équilibrée. En revanche, la bonne gouvernance concerne, en substance, la définition des objectifs (politiques ou économiques) du gouvernement et ses résultats. C'est ainsi que celle-ci s'inscrit souvent dans le courant " managérial ». L'objectif est alors d'introduire des préoccupations éthiques et démocratiques parmi les impératifs de gestion de l'entreprise ${ }^{94}$. Autrement dit, la notion de bonne administration se distingue de la bonne gouvernance par le truchement de la distinction entre les moyens et les résultats. Il peut en effet y avoir bonne administration et mauvaise gouvernance. En témoignent certaines dictatures aux moyens administratifs malheureusement très adaptés...

De surcroît, la bonne gouvernance ne semble pas obéir à une logique de pondération ou encore de modération du droit : la corruption « est ou n'est pas ». C'est pourquoi, à notre sens, les questions de transparence, de participation, d'accessibilité et de responsabilité de l'administration relèvent davantage de la bonne gouvernance que de la bonne administration. Plus exactement, elles

92 M.-L. Bemelmans-VideC, "Guidelines and Standards: Assuring the Quality of Evaluation and Audit Practice by Instruction", in Robert Schwartz and John Mayne (ed.), Quality Matters. Seeking Confidence in Evaluating, Auditing, and Performance Reporting, New Brunswick: Transaction Publishers, 2005, pp. 151-152, 166.

93 ELOI DiaRRA, «Les administrés et l'administration », in Laurence Burgorgue-Larsen (dir.) La France face à la Charte des droits fondamentaux de l'Union européenne, Bruxelles: Bruylant, 2005, p. 605 . 
sont la transposition, sur le plan administratif, de l'idée de démocratisation des rapports entre les citoyens et l'État.

Du fait de sa distinction avec la bonne gouvernance, la notion de bonne administration renforce son contenu spécifique. S'il ne faut donc pas assimiler ces deux notions, il ne faut pas non plus nier leur lien évident. La bonne administration peut donc être intégrée dans un objectif plus large de bonne gouvernance. Le rapport entre ces notions est à l'image de l'enrichissante interdisciplinarité entre le droit et les autres domaines, notamment le domaine politique. De plus, la définition de la bonne administration ici proposée peut heureusement faire l'objet de futures discussions ou critiques, qui relativiseraient alors la différence établie ici entre les deux notions.

\section{APPEL AU DÉBAT CONCEPTUEL}

La définition à donner à la " bonne administration " en droit nous semble être un débat utile ne se cantonnant pas à de pures réflexions théoriques. Le véritable souci de l'effet concret d'une norme passe en effet par la définition de son contenu. Loin de considérer que notre proposition de définition devrait être exempte de critiques, nous appelons donc simplement à la discussion.

À titre d'exemple, la définition ici proposée (adaptation équilibrée des moyens de l'administration publique) ne se confond-elle pas avec celle de proportionnalité ? Toutes deux partagent en effet à la fois les idées d'adaptation (ou d'adéquation) et de pondération. De plus, la proportionnalité invite, à l'instar de la bonne administration, à une analyse affinée du fonctionnement administratif et s'inscrit dans une logique dialectique. Mais si ces notions sont liées, elles demeurent toutefois, à notre sens, indépendantes, pour au moins trois raisons. D'abord, la proportionnalité couvre un domaine plus étendu que la bonne administration. Celle-ci est, comme nous l'avons déjà indiqué, cantonnée à l'administration publique. Ensuite, si la proportionnalité relève du « raisonnement dialectique associé à des droits dits fondamentaux $»^{95}$ et, plus généralement, des limites de ces droits, cette idée d'atteinte n'est pas une condition d'apparition de la notion de bonne administration. Enfin, l'idée de pondération, commune aux deux notions, ne porte néanmoins pas sur le même objet. Alors que la pondération désigne, dans le cadre de la notion de bonne administration, la pertinence de plusieurs éléments, dans le cadre de la notion de proportionnalité, elle renvoie au rapport de concordance entre deux éléments (l'objectif et l'atteinte au(x) droit(s)). 
L'évaluation de l'importance de l'objectif poursuivi, qui doit alors entretenir un rapport raisonnable avec l'importance de l'atteinte au droit est incluse dans la proportionnalité tandis qu'elle est exclue de la notion de bonne administration, telle que nous l'avons définie.

De nouveau, une autre conception - extensive - est possible. C'est d'ailleurs celle adoptée par la majorité des rédacteurs de texte de droit. Il n'est donc pas surprenant que bon nombre de juristes associent intuitivement la « bonne administration " à un agrégat de principes ou de notions. Mais dans ce cas, pourquoi ne pas justifier davantage cette approche?

À l'inverse, et si tant est que nous convainquions sur la nécessité de définir la «bonne administration » de manière restrictive, de multiples sens pourraient lui être conférés au sein d'un même système, et selon les cultures juridiques. Nous n'appelons donc pas tant à l'acceptation unanime de notre définition, mais simplement à un débat conceptuel lui-même né du droit positif ; autrement dit, à un échange dépassant tout type de frontières...

\section{BIBLIOGRAPHIE}

Académie FrançaISE. Dictionnaire de l'Académie française, fasc. II, 9. éd., Paris : Imprimerie nationale, 1987.

Alonso Mas, Doña María José. La solución justa en las resoluciones administrativas, Valencia: Tirant lo Blanch, 1998.

Aristote. Éthique à Nicomaque, Paris : J. Vrin, 1987.

Aubenque, Pierre. La prudence chez Aristote, Paris : P.U.F., 1997.

Auby, Jean-Bernard, et Dutheil de la Rochère, Jacqueline (dirs.). Droit administratif européen, Bruxelles : Bruylant, 2007.

AzOULAI, LoÏC. "Le principe de bonne administration », in Jean-Bernard Auby et Jacqueline Dutheil de la Rochère (dir.), Droit administratif européen, Bruxelles : Bruylant, 2007, pp. 235-241.

Bemelmans-VIDec, M.-L., "Guidelines and Standards: Assuring the Quality of Evaluation and Audit Practice by Instruction", in Robert Schwartz and John Mayne (ed.), Quality Matters. Seeking Confidence in Evaluating, Auditing, and Performance Reporting, New Brunswick: Transaction Publishers, 2005.

Bentham, Jérémie. Déontologie ou science de la morale, Paris : Charpentier, 1834.

Bevir, Mark. "What is Governance", in Mark Bevir (dir.), Public Governance, t. 1, London: Sage Publication, 2007. 
Bousta, RhiTA. "Contribution à une définition de l'Ombudsman ", RFAP, n. ${ }^{\circ} 123$, Paris : Ecole nationale d'administration, 2007.

Bousta, RHITA. "Droit des étrangers - Mais à quoi sert le droit à une bonne administration? ", Revue des droits de l'Homme, n. ${ }^{\circ}$ 12, Avignon : OpenEdition, 2017, http:// revdh.revues.org/3216.

Bousta, RHITA. Essai sur la notion de bonne administration en droitpublic, Paris : L'Harmattan, 2010

Bousta, RHITA. "Who said there is a right to good administration? A critical analysis of art. 41 of the CFreu", European Public Law Review, vol. 19, n. ${ }^{\circ}$ 3, Alphen aan den Rijn: Kluwer Law International, 2013.

Bouvier, Michel. "Avant-propos », in Michel Bouvier (dir.), Réforme des finances publiques, démocratie et bonne gouvernance, Paris : L.G.D.J., 2004

Cabanes, Arnaud. Essai sur la gouvernance publique. Un constat sans concession... quelques solutions sans idéologie, Paris : Gualino, 2004.

CaIllosse, JACQUES. La constitution imaginaire de l'administration. Recherches sur la politique du droit administratif, Paris : P.U.F., 2008.

Callaghan, A. J. "Maladministration", The Ombudsman Journal, n. ${ }^{\circ}$, Vienna: International Ombudsman Institute, 1988

Chevallier, JACQues. " De l'administration démocratique à la démocratie administrative », R.F.A.P., 1/01/2011, n. ${ }^{\circ} 137-138$, pp. 217-227.

Chevallier, Jacques. L'État post-moderne, 2. éd., Paris : L.G.D.J., 2004.

Chevallier, Jacques. La notion du nécessaire chez Aristote et chez ses prédécesseurs, Paris : Librairie Félix Alcan, 1915.

Chevallier, Jacques et Danièle Loschak. "Rationalité juridique et rationalité managériale dans l'administration française ", R.F.A.P., n. ${ }^{\circ} 24$, Paris : Documentation Française, 1982.

Constantinesco, LéOntin-Jean. Traité de droit comparé. La méthode comparative, t. 2, Paris : L.G.D.J., 1974

Cornu, Gérard. Vocabulaire juridique, 8. éd., Paris : P.U.F., 2007.

De Waard Boudewijn, W. N., et Adrienne J. C. De Moor-van Vugt. « Les changements récents dans le droit administratif néerlandais ", in Gérard Marcou (dir.), Les mutations du droit de l'Administration en Europe. Pluralisme et convergences, Paris : L'Harmattan, 1995. 
Dellis, GeORGES. « Le principe de bonne administration dans la jurisprudence du Conseil d'Etat hellénique ", in Mélanges en l'bonneur de Epaminondas P. Spiliotopoulos. Etat-loi-administration, Bruxelles : Bruylant, 1998.

Delmas-Marty, Mireille. Les forces imaginantes du droit, t. 3, La Refondation des pouvoirs, Paris : éd. du Seuil, 2007.

Diarra, EloI. « Les administrés et l'administration », in Laurence Burgorgue-Larsen (dir.), La France face à la Charte des droits fondamentaux de l'Union européenne, Bruxelles : Bruylant, 2005.

Echaudemaison, C.-D. Dictionnaire d'Économie et de Sciences sociales, 6. ${ }^{e}$ éd., Paris : Nathan, 2003.

FALzONE, Guido. Il dovere di buona amministrazione, Milan: Dott. A. Giuffrè, 1953.

Farazmand, Ali. "Sound Governance in the Age of Globalisation: A Conceptual Framework", in Ali Farazmand (dir.), Sound Governance, London: Praeger, 2004.

FERnÁNDEZ FARRERES, GERmÁn. La subvención: concepto y régimen jurídico, Madrid: Instituto de Estudios Fiscales, 1983.

García de Enterría, Eduardo. "La interdicción de la arbitrariedad en la potestad reglamentaria", R.A.P., n. ${ }^{\circ}$ 30, Madrid: Centro de Estudios Políticos y Constitucionales, 1959.

Gaudin, Jean-Pierre. Pourquoi la gouvernance ? Paris : Presses de science politique, 2002.

Gil-Robles Álvaro y Gil-Delgado. "La filosofía del defensor del Pueblo", in El procurador del común. Defensor del Pueblo y Comunidades Autónomas, Valladolid: Cortes de Castilla y León, 1995.

Gómez Puente, Marcos. La inactividad de la Administración, Madrid: Aranzadi, 2002.

Kortmann, COnSTANTin. "Les principes généraux du droit dans le droit public néerlandais », in Christian-Albert Garbar (dir.), Mélanges en l'honneur de Benoît Jeanneau. Les mutations contemporaines du droit public, Paris : Dalloz, 2002.

KuUsikKo, KIRSI. "Advice, Good Administration and Legitimate Expectations: Some Comparative Aspects", European Public Law, vol. 7, n. ${ }^{\circ} 3$, Alphen aan den Rijn: Kluwer Law International, 2001.

Lakehal, Mokhtar. Dictionnaire d'économie contemporaine et des principaux faits politiques et sociaux, 3. éd., Paris : Vuibert, 2000.

LASSALLE, JULIE. Le principe de bonne administration en droit communautaire, Thèse droit public, Paris : Université de Paris II, 2008. 
MARCOU, GÉRARD. "Gouverner les villes par le droit ? », C.U.R.A.P.P., La gouvernabilité, Paris : P.U.F., 1996.

Marcou, Gérard. "La Gouvernance : innovation conceptuelle ou artifice de présentation? ", Annuaire 2006 des Collectivités locales : La gouvernance territoriale, Paris : C.N.R.S., 2006.

Minorgue, Martin. "Is Good Governance a Universal Value?", Public Policy and Management: Working Paper Series, n. ${ }^{\circ}$ 6, Manchester: University of Manchester, 1998, p. 4

MORE, ThOMAS. Le traité de la meilleure forme de gouvernement ou l'Utopie, Genève : Librairie Droz, 1983 [1936].

Morvan, FrançOIS. "Les aspects majeurs de la nouvelle gouvernance financière ", in Michel Bouvier (dir.), Réforme des finances publiques, démocratie et bonne gouvernance, Paris : L.G.D.J., 2004.

Muzny, Peter. La technique de proportionnalité et le juge de la Convention européenne des droits de l'bomme. Essai sur un instrument nécessaire dans une société démocratique, Aix-en-Provence : P.U.A.M., 2005.

Nauwelaers, Michèle, et Olivier Dutheillet de Lamothe. A.J.D.A., chron., Paris : Dalloz, 1978

Nehl, Hanns Peter. Principles of Administrative Procedure in EC Law, Oxford: Hart Publishing, 1999

Nieto, Alejandro. La "nueva" organización del desgobierno, 3. ${ }^{a}$ ed., Barcelona: Ariel, 2003.

Parejo Alfonso, Luciano. Crisis y renovación en el derecho público, Madrid: Centro de Estudios Constitucionales, 1991.

Pérez Calvo, Alberto. "Aspectos constitucionales del Defensor del Pueblo", Revista del Departamento de Derecho Político, n. ${ }^{\circ} 4$, Madrid: UNED, 1979.

Pollitt, Christopher, Sandra van Thiel and Vincent Homburg. New Public Management in Europe. Adaptation and Alternatives, Basingstoke: Palgrave Macmillan, 2007.

Pollitt, Christopher, Sandra Van Thiel and Homburg, Vincent. New Public Management in Europe. Adaptation and Alternatives, Basingstoke : Palgrave Macmillan, 2007.

Ponce Solé, Juli. Deber de una buena administración y derecho al procedimiento administrativo $y$ del ejercicio de la discrecionalidad, Valladolid: Lex Nova, 2001.

Pontier, Jean-Marie. "Qu'est-ce que le droit administratif ? », A.J.D.A., n. ${ }^{\circ}$ 35, Paris : Dalloz, 2006. 
Rials, StÉPHANE. Le juge administratif français et la technique du standard. Essai sur le traitement juridictionnel de l'idée de normalité, t. 135, Paris : L.G.D.J., 1980.

Runavot, Marie-Clothilde. « La bonne administration : consolidation d'un droit sous influence européenne », R.F.D.A., 1/03/2010, n. ${ }^{\circ} 2$, pp. 395-403.

Sabourin, Paul. "Recherches sur la notion de maladministration dans le système français », A.J.D.A., 20 septembre 1974, pp. 396-407.

Schwarze, Jürgen. European Administrative Law, London: Sweet \& Marwell, 1992.

Simon, Herbert A. Administrative Bebavior. A Study of Decision-Making Process in Administrative Organization, New York: The Free Press, 1957.

SÖDERMAN, JACOB. " Le citoyen, l'administration et le droit communautaire », R.M.U.E., n. ${ }^{\circ}$ 2, Paris : Clement Juglar, 1998

Stephanou, Constantin A. " La nouvelle gouvernance européenne : bilan et perspectives ", Cabiers de droit européen, vol. 42, n. ${ }^{\circ}$ 5-6, Brussels : Léon Goffin, 2006.

StOker, Gerry. "Cinq propositions pour une théorie de la gouvernance », Revue internationale des sciences sociales, n. $^{\circ}$ 155, Toulouse : ERES, 1998.

Stowe, Kenneth. "Good Piano won't play Bad Music: Administrative Reform and Good Governance", Public Administration, vol. 70, Nueva Jersey: Wiley-Blackwell, 1992.

TOMÁs Mallén, Beatriz. El derecho fundamental a una buena administración, Madrid: Instituto Nacional de Administración Pública, 2004.

Uría FernándeZ, Francisco. "El buen gobierno en al ámbito del sector público. Una reflexión jurídica", en Manuel Balmaseda y Melguizo et al. (dir.), La Administración pública que España necesita, Madrid: Círculo de Empresarios, 2007.

Vedel, GeORGES. "La juridiction compétente pour prévenir, faire cesser ou réparer la voie de fait administrative", J.C.P., vol. I, n. ${ }^{\circ} 851$, Paris : CFJ, 1950.

Wheare, Keneth Clinton. Maladministration and its remedies, London: Stevens \& Sons, 1973.

Woodhouse, Diana. In Pursuit of Good Administration. Ministers, Civil Servants and Judges, Oxford: Clarendon Press, 1997. 